\title{
La ruta del día a día: cotidianidad laboral en una empresa de transporte público urbano en el Perú neoliberal
}

\begin{abstract}
Alejandro Villanueva Gutiérrez
Estudiante de Antropología en la Pontificia Universidad Católica del Perú (PUCP). e-mail: a20155484@pucp.edu.pe
\end{abstract}

\section{Resumen}

La aplicación de políticas neoliberales a partir de la década de 1990 implicó reformas en el sector de transporte. Estas reformas significaron la liberalización del sistema de transporte, con las cuales se eliminaron varias restricciones y se limitó la intervención estatal. Esto tuvo repercusiones en el funcionamiento de las empresas, como es en el ámbito laboral. Para ilustrar los efectos de tales medidas, se toma como ejemplo el caso de la empresa Real Express presentando a sus actores y funciones respectivas como una aproximación a su funcionamiento. Sobre este, resaltan condiciones de precariedad laboral, frente a las cuales surgen mecanismos de resistencia por parte de trabajadores a través de una economía moral $\mathrm{y}$ otras estrategias individuales que les permiten adaptarse a tales condiciones y maximizar sus beneficios en cuanto les sea posible. Así, se concluye que frente a las adversidades que puedan afrontar los trabajadores de tal empresa, pueden adecuarse a tales situaciones y aminorar los perjuicios respaldándose en diversos recursos sociales e individuales que puedan disponer.

\section{Palabras clave}

Neoliberalismo, transporte público urbano, cadena de valor, precariedad laboral, resistencia, economía moral. 


\title{
The day-to-day route: daily work in an urban public transport company in neoliberal Peru
}

\author{
Alejandro Villanueva Gutiérrez \\ Anthropology student at the Pontifical Catholic University of Peru (PUCP). \\ e-mail: a20155484@pucp.edu.pe
}

\begin{abstract}
The application of neoliberal policies from the 1990s implied reforms in the transport sector. These reforms meant the liberalization of the transportation system, with which various restrictions were removed and state intervention was limited. This had repercussions on the operation of companies, as occurs in the labour sphere. To illustrate the implications of such state measures, the company Real Express is taken as an example, of which the respective actors and functions are presented as an approach to the development of their activities. Out of this functioning, conditions of labour precarity stand out, in the face of which mechanisms of resistance by workers against such arise through a moral economy and other individual strategies that allow them to adapt to such conditions and maximize their benefits as much as possible. Thus, it is concluded that in the face of the adversities that the workers of that a company may face, they can adapt to those situations and lessen the damages, based on various social and individual resources that they may have.
\end{abstract}

\section{Keywords}

Neoliberalism, urban public transport, value chain, labour precarity, resistance, moral economy 


\section{Introducción}

El transporte público es parte de la cotidianeidad de la gran mayoría de personas en los centros urbanos del Perú, sobre todo en Lima. Así, es común escuchar comentarios en los cuales se critica abiertamente el servicio ofrecido por los transportistas; comentarios entre los que destacan la imprudencia de los conductores por exceso de velocidad o, su contraparte, la lentitud de los vehículos en llegar a su destino; el mal estado de los vehículos; reclamos por cobros considerados injustos; entre otros. Pero poco se conoce sobre el "otro lado" del transporte; esto es, sobre quienes brindan tal servicio y bajo qué condiciones lo hacen. Por lo tanto, antes de profundizar sobre estos aspectos, es importante resaltar de manera breve el proceso histórico a partir del cual surgió este servicio.

Una investigación realizada por el Instituto de Libertad y Democracia (ILD) en el año 1990 propuso cuatro etapas del transporte urbano en Lima (citado en Guillermo \& Tello, 2018, p. 32). Una primera etapa (1874-1921) y formativa del transporte en Lima, que se caracterizó por la transición de la tracción animal a la fuerza eléctrica, y la consecuente aparición de tranvías y trenes. La segunda etapa (1921-1930) se caracteriza por la introducción de los primeros ómnibus, los cuales eran operados por concesionarias privadas, llegando a ser ocho empresas las prestadoras de servicios para 1926. La tercera etapa (1931-1965) fue en la que, según los investigadores, colapsó el transporte tradicional y emergió, predominantemente, el informal (Guillermo \& Tello, 2018, p. 33); además, tuvo lugar la crisis de transportistas formales en los años cincuenta, que desencadenó una serie de quiebras de empresas de transporte a partir de 1959. Por último, en la cuarta etapa (de 1965 en adelante), se cancelaron las concesiones a las empresas privadas quebradas, que contaban con omnibuses y rutas limitadas, y fueron reemplazadas con rutas más diversas de acuerdo a la demanda popular, por lo que se tuvo que recurrir al uso de microbuses. Así, hacia 1990, era tan grande y desbordante la demanda que, según ILD, más del 95\% de líneas de transporte no funcionaban de manera legal (Guillermo \& Tello, 2018; Bielich, 2009).

Sin embargo, la investigación del ILD considera solo las etapas previas a 1990. Por ello, para fines explicativos, se propone aquí una quinta etapa, en la cual se toma como fecha de inicio el año 1991 y continúa en vigencia hasta la actualidad. Esta etapa inició en un contexto de cambios estructurales, de carácter político-económico, en el país. Un punto de partida fue la aplicación definitiva del programa del Consenso de Washington por parte del gobierno del recién electo presidente, Alberto Fujimori, en el que se fomentó la apertura del Estado para el ingreso de capital extranjero, con el fin de situar al mercado internacional como el proveedor principal de recursos, limitando la intervención fiscalizadora del gobierno en las empresas y servicios, bajo el principio de la autorregulación del mercado (Jiménez, 2001, p. 146). Pero, de facto, esta quinta etapa iniciaría en julio de 1991 con la promulgación del Decreto Legislativo $N^{\circ} 651$, en el cual se implementó la liberalización del transporte a través 
de la aplicación de una serie de medidas, tales como el establecimiento de la libre competencia de tarifas en el servicio; el libre acceso a rutas; la nueva posibilidad de que personas naturales o jurídicas prestasen el servicio; y la otorgación de permisos para cualquier vehículo de brindar el servicio. Otro detalle de este decreto es que estableció la eliminación de restricciones sobre la importación de vehículos usados al país. La promulgación de esta ley se podría considerar como parte del proceso de privatización de servicios públicos durante el gobierno fujimorista, en el cual el Estado, desde entonces, dejó de gestionar el transporte para dejarlo a potestad de las empresas privadas (Bielich, 2009, p. 29).

Esta serie de políticas implementadas de corte neoliberal permite entender varias particularidades del funcionamiento integral del sistema de transporte. En primer lugar, a partir de los antecedentes históricos, estas políticas surgen en un contexto en el cual casi todas las líneas de transporte eran informales ${ }^{1}$. A su vez, a partir de la instauración de tales medidas, se estimuló la compra de vehículos del exterior (estén en buenas o malas condiciones) y el uso de diversos tipos de vehículos para emplearse en el servicio de transporte; se flexibilizaron tanto la propiedad sobre las rutas por parte de pocas personas, como las condiciones laborales de los trabajadores. Sin embargo, como se verá en este caso, estas condiciones laborales adversas no iban a ser aceptadas como inexpugnables por los/as trabajadores/as, sino que, al contrario, generarían mecanismos a manera de adaptación frente a ellas.

De esta manera, la presente investigación propone brindar una aproximación al funcionamiento del transporte público urbano, tomando como principal referencia un caso etnográfico sobre los trabajadores de la empresa Real Express; a partir de este funcionamiento demostrar cuáles son las condiciones adversas y cómo se manifiestan, para finalmente poder explicar los mecanismos de adaptación frente a tales. Este estudio etnográfico es producto de una breve investigación, realizada entre los meses de octubre y noviembre del año 2018, llamada La autarquía de los transportistas. Informalidad dentro de la formalidad en una empresa de transporte público urbano ${ }^{2}$, realizada como trabajo grupal para el curso de Antropología Urbana en la Pontificia Universidad Católica del Perú. Durante el trabajo de campo, se pudo recorrer las rutas de la empresa con los transportistas e ingresar a los terminales de vehículos, en los cuales se aplicaron técnicas como la observación participante y entrevistas semi-estructuradas; estas últimas realizadas a quince conductores, a cinco cobradoras, un cobrador y una despachadora. Como resultado su aplicación, se logró conocer cuáles eran los actores implicados y sus funciones dentro de la empresa. Así, luego de haber identificado a los actores de interés, se les pudo categorizar en

\footnotetext{
1 La informalidad se entiende en el presente texto como la falta de reconocimiento legal por parte del Estado y, por consecuencia, poca o nula capacidad de intervención y regulación en tal esfera.

2 Los miembros del grupo que formaron parte de este trabajo son Gonzalo Zubiaurr, Isabel Chiri, Alejandro González, Andrea Rentería y yo, Alejandro Villanueva. Los miembros tienen pleno conocimiento y aceptación de la realización de este artículo.
} 
cuatro tipos $^{3}$ : principales, secundarios, terciarios y externos -conforme al enfoque del estudio. Los principales son los choferes; los secundarios, los cobradores; los terciarios se dividen en dos tipos: directos (agentes fiscalizadores) e indirectos (junta directiva); y, finalmente, los externos son los dateros. Cabe recalcar que en la investigación figuran los nombres reales de quienes fueron informantes y se cuenta con el pleno conocimiento y consentimiento de estas personas sobre el manejo de la información para el desarrollo del presente artículo.

La investigación se divide en dos secciones: la primera se desarrollará tomando como base los datos etnográficos obtenidos y se realizará la presentación de los actores y la cadena de valor surgida a partir de la interacción entre estos. El enfoque de cadena de valor se entiende, desde la propuesta de Kaplinsky y Morris, como el rango de actividades requeridas para desarrollar un producto o servicio a través de sus diferentes fases de producción hasta su consumo (2000, p. 4). Esto implica incluir al total de actividades y fases de producción, las cuales solo se entienden a partir de los actores que intervienen. Mientras que el trabajo de Supervielle y Rojido propone que este enfoque invita a reflexionar sobre la relación entre diferentes actores de acuerdo a sus actividades particulares y su posición dentro del proceso productivo (2007, p. 13), resaltando la interconexión existente entre los actores. Así, se podrá lograr un acercamiento, a partir de ambos aspectos, sobre las condiciones laborales en tal empresa.

En la segunda sección, se desarrollará lo referido a la resistencia de los/as trabajadores/ as en el transporte público urbano. Para esto, primero, se introduce al tema desde el concepto de precariedad laboral, del cual uno de los principales aportes para este trabajo es el de Suliman y Weber (2019), quienes la definen a partir de sus características, que son la falta de garantía de empleo de largo plazo, inseguridad en la tenencia de provisiones necesarias para el bienestar de los trabajadores, como sería a través de prestaciones de salud o sistemas de pensiones (2019, p. 535). De este modo, la precariedad se define por sus carencias de beneficios laborales, lo cual contrasta con otros empleos que sí puedan aprovechar de tales condiciones. Está además el aporte de Cabrales, en el cual se indica que la precariedad laboral surge como consecuencia del funcionamiento de prácticas político-económicas propias del neoliberalismo, en las cuales el crecimiento económico está ligado a un reemplazo de la seguridad laboral por un trabajo flexible, lo que responde al principio de maximización de utilidades, de modo que deja de garantizar condiciones laborales óptimas para el desempeño de trabajadores (2011, p. 55). Por último, desde otra mirada al concepto, se incluye la propuesta de Kasmir, quien resalta la la precariedad desde la experiencia humana. De esta manera, ofrece una mirada del fenómeno menos desde las relaciones de clase y se enfoca más en los sentimientos de

\footnotetext{
3 Los criterios para la categorización fueron los siguientes: el nivel de visibilidad pública (de mayor a menor) y de interacción con los demás actores. Mientras que los principales y secundarios son la cara visible de la empresa y prestadores directos del servicio a los clientes, los terciarios cumplen funciones administrativas-fiscalizadoras, y los externos dependen de los principales para intervenir en el circuito.
} 
vulnerabilidad, desplazamiento y desesperanza (2018, p. 2). Así, se agrega el factor de la experiencia constante de aquellas personas que se encuentran en tal situación (transversal a diferentes contextos) y permite entender cómo pueden actuar frente a esta. A manera de síntesis, una propuesta integral de la precariedad considerará las condiciones laborales adversas, el contexto político económico junto con sus lógicas y cómo estas se vuelven tangibles en la experiencia cotidiana de las personas de modo que puede tener cierta influencia en su comportamiento económico.

Es a partir de lo anterior que se puede explicar la resistencia, tomando como referencia los aportes de Giraldo y Del Valle, quienes plantean una definición basada en la propuesta conceptual de Foucault ${ }^{4}$. El primero, Giraldo, señala que este concepto no representa una imagen invertida del poder, sino que es "tan inventivo, móvil y productivo como él" (2006, p. 117); por esto, la resistencia funciona con la misma lógica que el poder, pero desplazándose de abajo hacia arriba, y se distribuye entre los actores (2006, p. 117). Con esto, resalta el carácter autónomo de la resistencia, la cual no se limita solo a contrarrestar el poder, sino que genera alternativas para sí, adecuándose al ejercicio del poder "desde arriba". Mientras que Del Valle, por su parte, destaca que la resistencia existe siempre que haya ejercicio de poder, por lo cual no puede existir poder sin su respectiva resistencia, ni resistencia sin relaciones de poder (2012, p. 162). Finalmente, Brown, en su propuesta crítica al concepto de resistencia, propone que, a pesar de la existencia de la dominación y subordinación, no es apropiado reducir a las complejas pugnas a una categoría conceptual, sino que debe considerarse que hay otras fuerzas sociales que funcionaban y funcionan independientemente de la resistencia (1996, p. 734). A partir de todas estas propuestas, se puede delimitar al concepto de resistencia para esta investigación como "la otra cara de la moneda" respecto al poder, de manera que actúa tan autónomamente como este; sin embargo, esta resistencia se desarrolla desde las características propias que tenga cada sociedad. Por lo tanto, se puede decir que puede existir resistencia en distintos contextos, pero que cada una de ellas opera de distinta manera, justamente a raíz de las particularidades de cada uno.

Y, por último, para desarrollar la noción de economía moral se emplearán tres aportes. Rebón, Kasparian y Hernández la explican como la valoración social particular que se da a determinadas prácticas económicas, de modo que las puede legitimar de acuerdo a si se consideran correctas o no para ese contexto específico (2015, p. 178-179). El segundo aporte es el de Vizcarra, quien la define como el equilibrio entre una racionalidad económica capitalista y comportamientos emotivos basados en obligaciones morales y normas sociales (2018, p. 40-43). Así, manifiesta la coexistencia entre diferentes valoraciones relacionadas a la economía, la cuales pueden generar prácticas que no sean acordes con las que la ideología económica prominente estipule. Finalmente, Palomera y Vetta complementan la propuesta anterior, indicando que la economía moral no implica, necesariamente, una lógica

\footnotetext{
No hay algún texto en específico de Foucault en que se desarrolle tal concepto, sino que se presenta a través de varias de sus obras.
} 
opuesta a la de mercado (2016, p. 420-421); así, surge la posibilidad de que la economía moral sea funcional a la economía capitalista, aunque provenga de un pacto social con ética económica distinta. Posteriormente, se explicará cómo esta economía moral se puede entender como parte de la resistencia antes mencionada.

\section{Cadena de valor en el transporte público urbano: caso Real Express}

La empresa de transporte seleccionada para la investigación etnográfica fue Real Express. Esta empresa es relativamente nueva -inscrita en el año 2010 y con inicio de sus actividades en $2011^{5}$ - y se ha constituido como sucesora de la empresa Orión: después de la polémica suscitada por los múltiples accidentes automovilísticos en los que estuvo involucrada, vehículos de tal empresa circulan bajo otros nombres como Holrex, HRE Express, Holding y Real Express (El Comercio, 2015). Según manifestaba cada trabajador ${ }^{6}$ a quien se le preguntó, la empresa Real Express cuenta con dos rutas las cuales son la OM-19 y OM-37 cuyos recorridos se inician desde el distrito de Villa El Salvador hasta el Callao divergiendo en los paraderos iniciales, y algunas calles y avenidas del recorrido. Mientras que la primera ruta llega hasta el extremo sur de Villa El Salvador, la segunda llega a la zona centro del distrito. Ya expuestos los detalles generales de la empresa como institución, se procederá a presentar, a partir del enfoque de cadena de valor, a los actores, sus respectivas actividades y las relaciones que existen entre estos dentro del proceso productivo de este servicio.

En la investigación, se consideró, en un principio, como los dos actores principales a choferes y cobradores; sin embargo, durante el desarrollo de la misma, se tuvo que reformular tal posición y se optó por considerar como el actor principal al chofer, ya que es el único que siempre estuvo presente y es en torno a él que comienza a desarrollarse la cadena de valor. En cambio, el cobrador podría ser considerado como prescindible, puesto que, en una gran mayoría de casos, los choferes eligen si desean trabajan en solitario o si necesitan solicitar un cobrador para el préstamo del servicio. Un tercer actor sería la misma empresa, la cual tiene es propietaria del derecho de la ruta y se materializa a través de dos tipos de actores: actores directos e indirectos.

Por el lado de los actores terciarios directos, se encuentran tres agentes fiscalizadores. Los primeros agentes fiscalizadores son los encargados de cobrar el derecho de ruta y son quienes, además, cada lunes entregan la tarjeta de control y los boletos que se darán a los clientes al momento en que usen el servicio. Un segundo agente fiscalizador es el/la despachador/a, quien se encarga de controlar la salida de los

\footnotetext{
5 Información obtenida a través de la página web de la SUNAT. Fuente: http://e-consultaruc.sunat.gob.pe/cl-ti-itmrconsruc/jcrS00Alias

6 Todos los trabajadores son considerados como actores en al artículo, pero se hará énfasis en los choferes.
} 
buses, así como de verificar que todos los trabajadores y vehículos cumplan con los requisitos necesarios -uniforme, documentos del vehículo, licencia de conducir, documentos de la empresa- para poder ejercer sus funciones. Los terceros agentes fiscalizadores no se encuentran en el terminal, sino en pleno camino de recorrido estos son los llamados relojes. Según comentaban los transportistas, los relojes son quienes se encargan de controlar la hora de paso por determinados paraderos cercanos al lugar de partida a través de la tarjeta de control. La finalidad del control establecido es que los buses no se chanten $^{7}$ en algún paradero y perjudiquen a otros transportistas de la misma línea. Finalmente, entre los actores terciarios indirectos se encuentra la junta directiva -compuesta por accionistas, gerente y dueño de la empresa. De ellos solo se supo a través de comentarios de los informantes, ya que se encuentran físicamente separados de los demás trabajadores: laboran únicamente en su oficina ubicada en el distrito de La Perla, Callao. Dicho esto, se procederá con la descripción de la cadena de valor en torno a las rutas de la empresa. Esta cadena de valor, como se mencionó previamente, vincula a actores, actividades y proceso de producción de una mercancía (Supervielle y Rojido, 2007), la cual es, en este caso, el servicio de transporte, desde los clientes hasta los dueños de la ruta. En otras palabras, la cadena de valor se articula a partir de las interacciones entre los diferentes actores y a través de las diferentes funciones que desempeñan. Sin embargo, para este caso etnográfico, la cadena de valor se ha limitado a las relaciones existentes entre quienes forman parte de esta empresa, dejando de lado algunos procesos que pueden estar vinculados como la producción de los vehículos y la interacción con los clientes.

La cadena de valor en esta empresa inicia desde los choferes. Estos pueden ser propietarios de los vehículos o en caso contrario pueden alquilarlo. Los vehículos disponibles para alquiler pueden ser vehículos de terceros o de la empresa, y los precios de alquiler se establecen dependiendo de la antigüedad y estado de los vehículos: oscilan entre un promedio de cien y ciento cuarenta soles por día. Los vehículos más costosos son los que pertenecen a la empresa y son modelos más modernos y con mayor capacidad para pasajeros. En lo que respecta a los ingresos recibidos, estos varían, también, de acuerdo a la cantidad de vueltas (una vuelta equivale a salida y retorno al lugar de partida) que realicen diariamente. Completando dos vueltas diarias en una semana se gana entre quinientos y setecientos soles, mientras que tres vueltas equivalen a un aproximado de mil soles -esto, suponiendo que el chofer trabaje solo-. En caso de trabajar junto a un cobrador, estas ganancias tienden a variar. En la mayoría de casos, el reparto depende más del chofer que del cobrador (usualmente con diferencia de veinte soles). Sin embargo, existen casos en que la repartición es equitativa. En los casos en que el reparto era equitativo, los choferes indicaron que ya habían tomado previamente parte del dinero para gastos

\footnotetext{
El término chantarse es una expresión que se usa para referirse al hecho de que un chofer estacione su vehículo en determinado paradero por un tiempo prolongado para poder recibir a una mayor cantidad de pasajeros, pudiendo perjudicar así a los otros vehículos de la misma línea que hayan iniciado su recorrido después de este.
} 
de combustible y los alimentos del día. También, es importante tener en cuenta que los gastos en reparación de vehículos, además de recaer enteramente en los choferes, implicaban, en algunos casos, perder días de trabajo. Por tales motivos, existía un mayor vínculo entre los choferes con los vehículos, a diferencia de los cobradores, quienes podían trabajar intermitentemente con uno o varios choferes.

Las siguientes relaciones económicas. que también son parte de la cadena de valor, se desarrollan con los agentes fiscalizadores. Primero, los choferes deben realizar un pago diario por derecho de ruta a los encargados de estos cobros que consiste de treinta y cinco soles diarios. En el caso de los días lunes, se genera un pago adicional de doce soles por la tarjeta de control y los boletos para pasajeros. Como fue explicado por Erika ${ }^{8}$, la despachadora, el pago por el derecho de ruta se realiza diariamente, a excepción de los días domingos y feriados, los cuales son considerados como días no laborables para la empresa, por lo que los agentes fiscalizadores no asisten a los locales y los transportistas se organizan autónomamente para establecer las horas de salida y el orden que seguirán para iniciar su ruta.

En el caso de los segundos agentes fiscalizadores, los despachadores, sus funciones se restringen al espacio del terminal de los vehículos. Como se mencionó anteriormente, su labor consiste en supervisar que los transportistas cumplan con los requisitos necesarios para que puedan ejercer sus funciones según las reglas de la empresa, además de controlar las horas de salida y llegada de los vehículos por medio de sus respectivas Tarjetas de Control, así como también avisar a los transportistas cuando ya es su turno de proseguir con su ruta. El pago a estos trabajadores es con un sueldo fijo y realizado por la misma empresa.

Durante el desarrollo de las rutas, aparecen los terceros agentes fiscalizadores, quienes son los relojes. Jesús Guerrero9 ${ }^{9}$, uno de los choferes de la empresa, pudo explicar con mayores especificaciones acerca del funcionamiento del trabajo de los relojes. Mencionó que desde el momento en que un vehículo sale del terminal e inicia su recorrido en la ruta, se comienza a contar el tiempo en que este tarda hasta llegar a determinados puntos específicos en la ruta en los cuales se encuentran los relojes a manera de control; sin embargo, si es que no llegan hasta cierta hora límite, se cobrará por parte de la empresa cinco soles por cada minuto de tardanza a partir de los dos minutos como multa por no haber cumplido con la hora obligatoria de llegada a tales paraderos. Para el caso de la línea OM-37, son tres relojes; para el de la OM-19, son cinco. De ahí en adelante en el recorrido, ya no hay más relojes; por ende, existe menor control sobre las horas de pasada. En ese momento, entra en juego otro actor, quien es externo a la empresa y cuya función depende más de su relación con los choferes. Estos son los dateros, cuya función consiste en anotar las

\footnotetext{
8 Cita extraída de La autarquía de los transportistas. Informalidad dentro de la formalidad en una empresa de transporte público urbano.

9 Cita extraída de La autarquía de los transportistas. Informalidad dentro de la formalidad en una empresa de transporte público urbano.
} 
horas de pasada de las diferentes líneas de microbuses e informar sobre esto a los choferes, de modo que ayudan a tener más controlada las horas en que pasaron otras líneas de la misma ruta.

A partir de lo descrito, cabe resaltar que son varios actores los que intervienen dentro de esta cadena de valor a través del cumplimiento de sus respectivas funciones. Sin embargo, por más equilibrados y fijos que puedan parecer los cobros y pagos, estos tienden a variar. Esto se debe a que, más allá de un comportamiento económico racional, hay factores culturales y contextuales que influyen en el comportamiento de las personas, sus relaciones interpersonales y, como consecuencia, en el dinero ganado.

\section{Resistencia de los trabajadores de Real Express}

Antes de proceder con la explicación sobre la resistencia y de qué manera surge en el contexto del transporte público urbano, es necesario presentar las condiciones económicas que caracterizan a tal empresa de transporte y cómo estas se enmarcan en el contexto político-económico. Previamente, se explicó cómo se articulan los diversos actores en la cadena de valor y de qué modo participan en el desarrollo del transporte público urbano -en este caso, de la empresa Real Express. Sin embargo, no se mencionó el rol de la junta directiva, conformada por los accionistas, el dueño y gerente de la empresa respecto sus funciones y cómo intervienen dentro de la cadena de valor. Por esto, si bien no se pudo entrevistar durante el trabajo de campo a tales funcionarios, resulta deducible el hecho de que las funciones administrativas son desempeñadas por ellos, pues todas las demás labores ya han sido copadas por los otros trabajadores. Además, es hacia ellos a los que dirigían la mayoría de ingresos de la empresa (derechos de ruta, alquiler de vehículos, tarjetas de control, boletos, entre otros), descontando los gastos por sueldos de algunos trabajadores.

Pero el obtener tales ingresos no es lo único que destaca a tales funcionarios -y, por ende, a la empresa, dada su condición de representantes- dentro de la cadena de valor. Como se explicó antes, el Estado, al dimitir en su rol como prestador de servicios de transporte, permitió que las empresas se desarrollen autónomamente -producto de la liberalización económica- y que no sean debidamente reguladas; por lo tanto, pueden existir casos en que no garantizan beneficios laborales para los trabajadores, pese a que se pueda contar con los recursos económicos para poder brindárselos.

Esta liberalización económica antes explicada, en lo que respecta al servicio de transporte público urbano, condujo a que personas específicas (accionistas), a través de la empresa como entidad privada, posean derechos de ruta. Por tal motivo, quienes deseen por trabajar en tales rutas deben pagarles para poder hacer uso de la ruta. De ese modo, se manifiesta una suerte de acumulación flexible, tal como la denominaría Harvey (1990), la cual implica un gran aumento del empleo en el sector de servicios -como es el caso del transporte urbano- y recurre a la flexibilidad de procesos 
laborales, de los mercados de mano de obra y de la misma producción -prestación del servicio-, permitiendo a empleadores determinar casi de manera absoluta las condiciones de los empleados (p. 170-173). Esta flexibilidad de procesos laborales y de los mercados de mano de obra, en la cual son los empleadores quienes tienen control sobre el trabajo de los empleados, permite un reemplazo sin dificultades, y no solo caracteriza a una acumulación flexible, sino también a una situación de precariedad laboral.

En el caso de Real Express, se puede evidenciar la falta de garantías laborales por la falta de contratos formales con los choferes y cobradores, la falta de gratificaciones y vacaciones, falta de seguros médico para trabajadores, entre otras carencias. De ese modo, se manifiesta la relación meramente informal entre la empresa y los trabajadores, en la cual cumplimiento de los requisitos (pago de derecho de ruta, uniforme, turnos de partida, vehículo registrado, entre otras) se convierte en la única condición para trabajar y no garantiza mayores beneficios, sino que todas las eventualidades surgidas durante las jornadas laborales son asumidas por los trabajadores.

En ese sentido, la cadena de valor en torno a la empresa Real Express manifiesta el funcionamiento de lógicas de mercado neoliberales mediante de la "maximización de utilidades" y la poca regulación y fiscalización del Estado sobre la empresa; específicamente, con respecto a las condiciones laborales y la cual se refleja en las limitadas garantías concedidas (rasgo de la informalidad) propias del trabajo flexible (Cabrales, 2011). Por esto, es importante tomar en cuenta que el proceso de formalización de las empresas se desarrolló, en algunos casos, en medio de un precario régimen laboral, que no fue supervisado por las autoridades gubernamentales, ni antes ni durante su funcionamiento.

Pese a la desfavorable situación de los trabajadores, no se genera una sumisión absoluta frente a estas eventualidades, sino que ellos son capaces de adaptarse, empleando diferentes mecanismos o estrategias que facilitan su adaptación frente a tales condiciones. Así, entra en juego el concepto de resistencia. De este modo, si bien el poder "desde arriba" puede ser considerado como el establecimiento de las condiciones laborales desfavorables generadas por las políticas económicas neoliberales y el accionar de la empresa, la resistencia -o poder "desde abajo"serían las estrategias que los transportistas afectados desarrollen para adecuarse y obtener, en la medida de lo posible, beneficios (Giraldo, 2006). Además, se pueden visualizar las relaciones de poder entre transportistas y empleadores. Mientras que estos últimos se basan en los marcos legales establecidos por el Estado para no garantizar condiciones laborales óptimas, constituyendo, así, el poder imperante; los transportistas contrarrestan ese poder a través de la resistencia por medio de diversos mecanismos que disponen bajo sus posibilidades, estableciéndose, de esta manera, el poder y su respectiva resistencia (Del Valle, 2012). Precisamente es esta resistencia la que se manifiesta a través de una economía moral e involucra a los diferentes actores quienes han intervenido directamente en la cadena de valor. 
Para este caso, la noción de economía moral se demuestra en una diversidad de ejemplos correspondientes a lo descrito por los informantes. Primero, la recurrencia de "favores" obtenidos por los transportistas al alquilar un vehículo de la empresa; seguido de los cariños $^{10}$ de cincuenta céntimos o un sol dados a los/as despachadores/ as para poder retornar a la ruta antes de la hora estipulada; las relaciones que puedan existir entre transportistas y los relojes, de modo que puedan reducir las multas por exceder tiempos; por último, como compensación por la información brindada y dependiendo del nivel de sus vínculos afectivos, los choferes ofrecen a los "dateros" mayores o menores retribuciones. En relación a lo anterior, los intercambios de favores entre diferentes actores configuran una "economía moral" que hace variable la circulación del dinero al nivel de las ganancias. Esta economía moral no se desarrolla negando un la racionalidad de la lógica de mercado, sino que todas esas negociaciones que implican obligaciones morales tienen como principio fines netamente económicos (Palomera y Vetta, 2016) (Vizcarra, 2018). En ese sentido, obtener simpatía de los despachadores implica tener la posibilidad de laborar más tiempo para poder obtener más dinero. Las diferentes estrategias desarrolladas por los diferentes actores de esta red económica tienen como finalidad minimizar costos y maximizar beneficios, completamente consecuente a la lógica de mercado.

Sin embargo, la resistencia no se desarrolla exclusivamente a través de la "economía moral", sino que existen, también, algunas estrategias empleadas por los mismos transportistas que son manifestaciones de tal resistencia. Algunos ejemplos serían los siguientes: "hacer no solo dos, sino tres recorridos en un día", trabajar seis o siete días en determinadas semanas, optar -en el caso de los choferes- por trabajar sin cobrador; corretear ${ }^{11}$ con otros vehículos o chantarse en un paradero estratégicamente, entre otros. Estas manifestaciones de resistencia no implican necesariamente una neutralización del poder que se ejerce sobre ellos, sino, más bien, una adecuación frente a tales situaciones desventajosas de modo que puedan desarrollar estrategias con la finalidad de maximizar los beneficios que puedan percibir. Si bien no pueden obtener, a través de la resistencia, los beneficios laborales para salir de su condición de precariedad laboral, si pueden optimizar sus beneficios monetarios como compensación ante la falta de los otros.

Así, se manifiesta lo propuesto por Brown (1996), quien señala que la resistencia no opera de manera aislada, sino en un contexto con determinadas fuerzas sociales preexistentes. Entre estas, algunas que han podido destacar son el consenso tácito que existe entre actores involucrados sobre la recurrencia a los cariños; la falta de regulación a lo largo de toda la ruta, lo que permitiría chantarse o corretear en partes del trayecto $\sin$ recibir sanciones (excepto en algunos casos en que reciben sanciones

\footnotetext{
10 El cariño es una jerga utilizada para referirse a dádivas monetarias que algunos transportistas entregan a otros de los operadores de la empresa como los "relojes" o despachadores para obtener simpatía y beneficios a cambio.

11 Corretear es otra de las jergas empleada por los transportistas que fueron entrevistados que refiere al competir con otros vehículos para llegar antes a los paraderos con la finalidad de recibir a los pasajeros antes que otros vehículo de la misma línea.
} 
morales por parte de otros transportistas); o, una de las más importantes, que no haya restricciones sobre la cantidad de vueltas y los días en que puedan hacerlas, con lo cual dependen más de ellos mismos. Por lo tanto, la resistencia pasa a constituirse como una conjugación entre la respuesta al poder y los recursos con que se disponga para materializar tal respuesta.

\section{Conclusión}

A manera de síntesis, es relevante resaltar a todos los actores y roles que intervienen en este contexto para comprender cómo se genera la resistencia de los trabajadores. Por un lado, a nivel de instituciones, el Estado se hizo presente (o ausente) a través de la reforma de transporte público, consecuente con las políticas neoliberales instauradas, las cuales se enfocaron en la privatización y liberalización del mercado hacia el exterior; sin embargo, no hubo interés en implementar una formalización integral de las empresas de transporte. Esto quedó demostrado en el caso de Real Express, cuya formalización se dio a nivel superficial sin considerar lo subyacente, dentro de lo que están incluidas las condiciones laborales respecto a los trabajadores (arreglo de autos descompuestos, falta de gratificaciones, vacaciones, seguro de salud y salario fijo establecido por la empresa).

De esta manera, es por medio de tales políticas que se genera un contexto en el cual los empleadores (dueños y accionistas de la empresa de transportes) ejercen poder sobre los trabajadores a través de los cobros permanentes, el constante control y falta de garantías laborales. Pero frente a tales adversidades, se facilita un ambiente propicio para la resistencia, la cual se desarrolla de manera individual en los trabajadores, pero se produce socialmente dada su legitimidad entre ellos. Así, indistintamente de cuántas y cuáles sean las adversidades, se enfocan en las formas existentes y los recursos sociales e individuales con que cuentan para poder adecuarse a tales condiciones, de modo que puedan maximizar sus beneficios hasta donde las limitaciones se los permitan. Con esto, se puede demostrar que, debajo de la superficie formal, que implica su reconocimiento legal por parte del Estado, opera una informalidad vinculada a condiciones estructurales, como las medidas gubernamentales, como también a distintas alternativas posibles frente a los vacíos dejados por un Estado y una empresa indiferentes. Si, a pesar del carácter "formal" de esta empresa frente al Estado, sus empleados/as están expuestos/as a tales condiciones de trabajo, es posible imaginarse en qué condiciones pueden encontrarse aquellos/as que incursionan en otros sectores laborales que continúan siendo informales. 


\section{Referencias bibliográficas}

Bielich, C. (2009). La guerra del centavo. Una mirada actual al transporte público en Lima Metropolitana. Lima: IEP. Recuperado de http://repositorio.iep.org.pe/bitstream/IEP/1007/2/ documentodetrabajo155.pdf

Brown, M. (1996). On Resisting Resistance. American Anthropologist, 98(4), 729-735. Recuperado de https://www-jstor-org.ezproxybib.pucp.edu.pe/stable/pdf/681880.pdf?ab segments $=0 \% 252 \mathrm{Fbasic}$ SYC-5152\%252Fcontrol\&refreqid=excelsior $\% 3 \mathrm{~A} 041 \mathrm{af} 5 \mathrm{bb} 74 \mathrm{e} 5 \mathrm{c}$ 4d571d454977b1e1da1

Cabrales, O. (2011). La precarización laboral y el desempleo como consecuencias del neoliberalismo y la globalización. Revista Tendencias \& Retos, (16), 43-57. Recuperado de https://dialnet.unirioja.es/servlet/articulo? codigo $=4929352$

Del Valle, N. (2012). Entre poder y resistencia. Tras los rastros de la política en Foucault. Revista Enfoques, 11 (17), 147-168. Recuperado de https:/dialnet.unirioja.es/descarga/ articulo/4127589.pdf

Giraldo, R. (2006). Poder y resistencia en Michel Foucault. Tabula Rasa, (4), 103-122. Recuperado de http://www.scielo.org.co/pdf/tara/n4/n4a06.pdf

Guillermo, L. y Tello, S. (2018). La regulación del transporte urbano en Lima: caso El Metropolitano (tesis de maestría). Universidad Peruana de Ciencias Aplicadas, Lima, Perú.

Harvey, D. (1990). La condición de la posmodernidad. Investigaciones sobre los orígenes del cambio cultural. Buenos Aires: Amorrortu editores S.A.

Jiménez, F. (2001). El modelo neoliberal peruano: límites, consecuencias sociales y perspectivas. En: CLACSO (Ed.), El ajuste estructural en América Latina. Costos sociales y alternativas (pp. 145-169), Buenos Aires: CLACSO.

Kaplinsky, R. y Morris, M. (2000). A handbook for value chain research. Inglaterra, Brighton: University of Sussex, Institute of Development Studies. Recuperado de https://www.researchgate.net/profile/Ahmad_Solgi/post/hi_can_any_one_help_ me_in_the_value_chain_analysis_of_timber_from_natural_forest_of_africa/ attachment/59d6254179197b807798394a/AS:317012738281472@1452593134531/ download/Handbook+for+value+chain+anlysis.pdf

El Comercio (2015, octubre 07) Orión: Lima desconocía que empresa ya opera con nuevo nombre. Recuperado de https:/elcomercio.pe/lima/orion-lima-desconocia-empresa-operanuevo-nombre-226418-noticia/

Palomera, J., y Vetta, T. (2016). Moral economy: Rethinking a radical concept. Anthropological Theory, 16 (4), 413-432.

Rebón, J., Kasparian, D. y Hernández C. (2015). La economía moral del trabajo. La legitimidad social de las empresas recuperadas. Trabajo y sociedad, (25), 173-194. Recuperado de https:// www.redalyc.org/pdf/3873/387341101010.pdf 
Suliman, S. y Weber, H. (2019). Global development and precarity: a critical political analysis. Globalizations, 16 (4), 525-540.

Supervielle, M. y Rojido, E. (Octubre de 2007). Trabajo de Organización y Cadenas de valor. Estado actual de la investigación sobre mundos y mercados de trabajo. Simposio llevado a cabo en el Primer Congreso Latinoamericano de Historia Económica, Buenos Aires, Argentina. Recuperado de http://www.audhe.org.uy/Jornadas_Internacionales_Hist_Econ/ CLADHE1/trabajos/Supervielle_Marcos_387.pdf

Vizcarra, S. (2018). La economía moral de la ilegalidad en la ciudad cocalera: significados y prácticas legitimadoras del narcotráfico en la ciudad de Pichari (2000-2017) (tesis de doctorado). PUCP, Lima, Perú. 